\title{
Autonomous and controlled regulation of performance-approach goals: Their relations to perfectionism and educational outcomes
}

\author{
Maarten Vansteenkiste $\cdot$ Stijn Smeets $\cdot$ \\ Bart Soenens · Willy Lens · Lennia Matos • \\ Edward L. Deci
}

Published online: 23 October 2010

(C) Springer Science+Business Media, LLC 2010

\begin{abstract}
The present research uses self-determination theory to examine whether autonomous and controlled regulation of performance-approach (PAp) goals would differentially predict educational outcomes and add to the variance explained by the goal strength. Two cross-sectional studies among 10th to 12th grade students supported this hypothesis but showed that when regulations of PAp goals were entered into the same regression equation with the PAp goal strength, the PAp goal strength no longer predicted outcomes. Study 2 examined perfectionism in relation to PAp goals and types of regulation, thereby showing that whereas adaptive and maladaptive perfectionism were both positively related to PAp goals, adaptive and maladaptive perfectionism were associated with autonomous and controlled regulations, respectively. Finally, path modeling indicated that autonomous and controlled regulations of PAp goals (but not PAp goals themselves) accounted for nearly all of the relation between the types of perfectionism and learning outcomes. The discussion emphasizes that it is critical to move
\end{abstract}

M. Vansteenkiste $(\bowtie) \cdot$ B. Soenens

Department of Psychology, Gent University,

H. Dunantlaan 2, 9000 Gent, Belgium

e-mail: maarten.vansteenkiste@ugent.be

\section{S. Smeets · W. Lens}

Department of Psychology, Catholic University of Leuven, Leuven, Belgium

L. Matos

University of Lima, Lima, Peru

E. L. Deci

Department of Clinical and Social Sciences in Psychology,

University of Rochester, Rochester, NY, USA beyond the consideration of PAp goals per se, thereby considering the types of regulation of PAp goals.

Keywords Performance-approach goals ·

Autonomous and controlled motivation .

Achievement goal theory $\cdot$ Self-determination theory

Some students like to perform well relative to their peers because trying to outperform others provides an enjoyable challenge to them. This goal of doing better than others may stimulate them to concentrate in the classroom, persist in the face of failure, and perhaps even achieve high grades. Other students feel pressured to score better than others, either because their parents, teachers, or other socializing figures pressure them to pursue this achievement goal or because self-worth concerns and perfectionist standards pressure them from within to pursue the goal.

A focus on obtaining higher grades than peers has been labeled a Performance Approach (PAp) goal (Elliot and Harackiewicz 1996) within the achievement goal literature (Elliot 2005; Kaplan and Maehr 2007). During the past few years, achievement goal researchers have been intensively debating whether and when the pursuit of PAp goals facilitates or detracts from self-regulated learning and achievement (Brophy 2005; Elliot and Moller 2003; Harackiewicz et al. 1998, 2002; Midgley et al. 2001; Senko et al. 2008). The present research contributes to this controversy by examining whether the autonomous and controlled regulations of PAp goals pursuits matter above and beyond the preditive value of the strength of pursuing PAp goals per se. Specifically, based on self-determination theory (Deci and Ryan 2000; Vansteenkiste et al. 2010), we suggest that regulating the pursuit of PAp goals for autonomous reasons will relate positively to self-regulated 
learning and achievement, whereas regulating the goals for controlled reasons will relate to more negative outcomes. In the second study we also examined adaptive and maladaptive perfectionism (Frost et al. 1990) as individualdifference variables being related to PAp goals and of the autonomous and controlled reasons for pursuing them. ${ }^{1}$

\section{Brief historical overview of the performance-approach goal controversy}

Achievement goal theory (Dweck 1986; Nicholls 1984), which has been concerned primarily with examining the antecedents and consequences of achievement goals, emerged from research on the achievement motive (McClelland et al. 1953) and the expectancy-value model of achievement motivation (Atkinson 1957; Atkinson and Feather 1966). Central to the achievement-goal theory has been the distinction between mastery and performance goals (Ames 1992; Dweck and Leggett 1988; Nicholls 1984). Mastery goals involve the aim of learning new material or improving one's skills. In contrast, the aim of performance goals is to perform better than others or avoid performing worse than others. ${ }^{2}$ According to Nicholls (1984), people with performance goals are ego-oriented: they either pursue feelings of self-worth and self-aggrandizement by scoring better than others or avoid feelings of inferiority and self-denigration by not performing worse than others (Butler 1992; Ryan 1982).

Initial work within achievement goal theory showed that the pursuit of mastery goals was associated with various positive outcomes, including intrinsic motivation, selfregulated learning, and deep-level learning, whereas performance goals were found to be positively related to surface processing but unrelated or negatively related to deep-level processing and self-regulated learning. Further, in some studies, performance goals were found to predict

\footnotetext{
${ }^{1}$ Although SDT uses the term "motive," as well as reasons and regulations, to refer to the distinction between autonomous and controlled motivations, we chose to use only the terms "reasons" and "regulations" here, because, within the achievement motivation tradition, the term motive has a different meaning. Motives refer to people's acquired, dispositional competence-based orientations, including the motive to succeed and the motive to avoid failure. In the hierarchical model of achievement motivation (Elliot and Church 1997), motives are hierarchically located as personality antecedents of achievement goals, with the pursuit of achievement goals being an expression of an underlying dispositional motive. The current research focused on students' autonomous reasons and controlled reasons for pursuing PAp goals and not on the dispositional motives that can energize the adoption of PAp versus other achievement goals.

2 Mastery goals and performance goals are sometimes referred to as task-involvement and ego-involvement, respectively, when discussed as states, while they are referred to as task goals and ego goals when discussed as individual differences (Nicholls 1984).
}

academic performance, but other studies failed to confirm these findings. Moreover, performance goals have been found to predict some negative learning-related outcomes, such as critical self-evaluation after failure (see Elliot and Moller 2003; Midgley et al. 2001 for reviews).

Attempting to reconcile the discrepant findings for performance goals, Elliot and Harackiewicz (1996) made a distinction between performance approach goals and performance avoidance (PAv) goals, a distinction reminiscent of Atkinson's (1957) distinction between the need for success and fear of failure. Elliot $(1999,2005)$ argued that competence can be valued as a positive outcome to be achieved or incompetence can be valued as a negative outcome to be avoided. Accordingly, PAp goals concern striving towards the attainment of normative competence, whereas PAv goals concern behaving to avoid normative incompetence. Elliot argued that PAp goals should yield positive effects, especially on achievement, because the external focus that characterizes these goals leads people to select strategic study strategies that yield high achievement. In contrast, PAv goals hinder learning and achievement, because the concern with failure is likely to disrupt the learning process, undermine the pleasure of learning, and result in lower performance. The bifurcation of performance goals into approach and avoidance goals appeared useful, as various subsequent studies convincingly showed that PAv goals, relative to both PAp and mastery goals, were related to more negative outcomes, including lower intrinsic motivation, lower self-efficacy, higher test anxiety, greater selfhandicapping, lower grades, and poorer psychological wellbeing (Elliot 1999, 2005).

Other research has examined the relations of PAp goals, relative to mastery goals, in the prediction of learning outcomes, and these findings are less clear-cut. In general, mastery goals, which are expected to lead individuals to become fully immersed in the learning activity, have been found to positively relate to a variety of positive affective and experiential outcomes such as intrinsic motivation, task-absorption, and psychological well-being. Furthermore, individuals with mastery goals have been found to report using deep-level cognitive strategies such as elaboration and organization and meta-cognitive strategies such as time management and monitoring (see Elliot and Moller 2003; Midgley et al. 2001 for reviews) and to be less likely to cheat (Anderman et al. 1998). However, the relations of mastery goals to achievement have been mixed with some studies finding non-significant relations (e.g., Elliot et al. 1999) and others finding positive relations (e.g., KenneyBenson et al. 2006; Matos et al. 2007). Mastery goals appear to be primarily predictive of achievement that reflects deep learning (e.g., Graham and Golan 1991) or achievement in unfamiliar activities (e.g., Vansteenkiste et al. 2007). Adopting a mastery orientation thus seems to 
enable people to effectively regulate their learning, to deeply process the learning material, and to derive a sense of inherent enjoyment from the learning, but it does not necessarily relate to superior performance.

As for PAp goals, studies have found them to be positively related to a number of positive outcomes and some negative outcomes, while being unrelated to a host of other outcomes and some of these associations have not been consistently replicated across studies (for a review see Elliot and Moller 2003). For instance, PAp goals have been positively related to positive outcomes such as academic self-concept, self-efficacy, effort-expenditure, grade aspirations, and graded performance, although the positive association with performance has not always been replicated (e.g., Lau and Nie 2008). Furthermore, compared to mastery goals, PAp goals are more likely to relate to the use of superficial rather than deep-processing strategies and are less likely to be related to intrinsic motivation, the use of meta-cognitive strategies, long-term retention, and psychological well-being. Finally, some studies found PAp goals to be related to negative outcomes such as anxiety, disruptive behavior, and low retention of knowledge (Midgley et al. 2001). Overall, the adoption of PAp goals seems related to a rather distinct pattern of outcomes: PAp goals seem to energize individuals to put more effort into their studying, resulting in higher grades. At the same time, a focus on outperforming others seems to relate to superficial and narrow-minded learning strategies that do not necessarily promote the experience of learning enjoyment.

This pattern of findings for PAp goals has provoked a controversy within the achievement goal literature (Brophy 2005; Harackiewicz et al. 1998, 2002; Midgley et al. 2001). Although it is clear that the pursuit of PAp goals is not invariantly positive, it is still not completely clear yet for which outcomes, under which circumstances, and for whom the pursuit of PAp goals is adaptive (Midgley et al. 2001). Accordingly, various suggestions have been made to sort out the confusing results (e.g., Barron and Harackiewicz 2001; Elliot and Moller 2003).

In this respect, it is important to note that Elliot and colleagues (Elliot 1999, 2005; Elliot and Moller 2003; Elliot and Murayama 2008) changed the definitions of mastery and performance goals, suggesting that the basis for feeling competent is different for the two types of goals. Mastery goals are pursued to develop and attain self- or task-referential improvement, such that competence at an activity is derived from and defined by improvement relative either to how one has done on the activity in the past or to the standards inherent in the task (Elliot 2005). In contrast, performance goals are pursued to attain and demonstrate performance that surpasses the performance of others or of normative standards, such that competence is derived from and defined by outperforming others.
Accordingly, PAp goals involve trying to do better than specific others or merely trying to do well in relation to a table of norms and PAv goals are aimed at avoiding doing worse than specific others or a normative standard.

With this new definition, self-esteem concerns are no longer an inherent component of performance goals. Elliot and colleagues argued that achievement goals need to be defined in terms of aims rather than reasons, and that the pursuit of these aims can be regulated by different reasons, one of which is self-esteem concerns (Elliot and Murayama 2008; Elliot and Thrash 2001) or what, in self-determination theory, is called controlled reasons. Indeed, Elliot (2005; see also Elliot and Thrash 2001) argued that PAp and PAv goals could be motivated by reasons as diverse as self-esteem concerns, self-presentation, challenge, social approval, and rewards. The crucial point is that what Elliot and colleagues refer to as self-esteem concerns-previously said to be the core element of the definition of performance goals (e.g., Nicholls 1984)—should no longer be considered an inherent part of the definition of performance goals (whether PAp or PAv); rather the definition of achievement goals should focus simply on the criteria (i.e., interpersonal, intrapersonal, or task-based) used to define competence.

The claim by Elliot and colleagues that the reason selfesteem concern should not be inherently part of the definition of PAp goals and that PAp goals can instead be viewed as being regulated by qualitatively different reasons set the stage for conceptualizations that could help gain insight into the pattern of outcomes associated with PAp goals. Specifically, it is possible that different reasons for pursuing PAp goals as aims may yield different associations with learning outcomes. Elliot and colleagues implicitly recognized this by arguing that the negative effects of PAp goals were primarily found in studies where self-esteem concerns were part of the operationalization of PAp goals (Elliot et al. 2008).

Building on the conceptual changes proposed by Elliot and colleagues, Urdan and Mestas (2006) recently tried to shed light on the different reasons for pursuing PAp and PAv goals. They interviewed 53 high school seniors who strongly endorsed performance goals and discovered that their reasons for pursuing PAp and PAv goals could be located into one of four categories (viz., appearanceapproach, appearance-avoidance, competition-approach, and competition-avoidance). Although Urdan and Mestas's (2006) research helped enrich our understanding of the types of PAp goals, their approach did not include direct questionnaire-based assessments of the reasons for pursuing PAp goals.

Herein, we suggest that it would be useful to apply the autonomous-controlled motivation distinction proposed in self-determination theory (SDT; Ryan and Deci 2000, 
2006) to the pursuit of PAp goals for two reasons. First, we expected the reasons (i.e., regulations) for pursuing PAp goals to explain additional variance in the outcomes beyond the strength of PAp goals themselves. Second, we expected autonomous and controlled reasons to pursue PAp goals to relate differently to learning and achievement outcomes.

\section{Autonomous and controlled regulation of performance-approach goals}

SDT distinguishes between being regulated by autonomous reasons and controlled reasons. Autonomous regulation involves acting with a full sense of volition or willingness. There are two bases for autonomous regulation: intrinsic motivation and well-internalized extrinsic motivation. In the case of intrinsic motivation, people find the activity or goal to be interesting and enjoyable. Applied to the issue of PAp goals, some individuals find the pursuit of PAp goals interesting, challenging, and enjoyable in its own right. Well-internalized extrinsic motivation (e.g., identification) concerns the personal endorsement of the behavioral regulation or goal because the activity or goal is of personal value. That is, individuals who pursue PAp goals for identified reasons experience outperforming others as personally important and valuable, for instance, because it serves the attainment of a long-term plan such as gaining admission to medical school. Because both intrinsic and identified regulations involve experiences of choice and volition, they are often combined under the umbrella of autonomous motivation (e.g., Vansteenkiste et al. 2004).

In contrast to autonomous regulation, controlled regulation pertains to feeling pressured to perform a behavior (Deci and Ryan 2000) or pursue a goal (Sheldon 2002). Two types of controlled regulation have been differentiated: external and introjected regulation. In the case of external regulation, people are engaging in an activity or pursuing a goal to meet external demands, avoid punishment, or obtain a reward. For instance, some students might be focused on obtaining better grades than others because their parents had promised them rewards for being among the top performers in their classes. Individuals can also pressure themselves, for instance, by buttressing their goal pursuit with threats of shame and guilt for poor performance and promises of self-aggrandizement and pride for good performance. To illustrate, some students feel really bad about themselves when they do not outperform their peers, and they feel a sense of self-importance when they do better than others. Their PAp goal-pursuit is thus regulated by introjected forces within themselves that are experienced as stressful and pressuring. Because external regulation and introjection are both experienced as pressuring, demanding, and alien to the self, they are often combined to form a controlled regulation composite (e.g., Vansteenkiste et al. 2005).

Since Ryan and Connell's (1989) first examination of elementary school students' autonomous versus controlled reasons for studying, dozens of studies have applied SDT's conceptual differentiation within the educational domain (see Reeve et al. 2004 for a review). The studies have shown clearly that autonomous regulation is associated with such positive outcomes as concentration, persistence, time management, and deep learning. In contrast, controlled motivation has predicted negative outcomes such as maladaptive coping strategies, test anxiety, superficial learning, and school dropout. These results have been obtained across age groups (i.e., from elementary to high school) and across cultures (e.g., Belgium, Canada, China, Japan, Russia, the U.S.). The distinction between autonomous and controlled reasons for studying has thus proven useful in predicting learning and achievement. Herein, we suggest that examining autonomous and controlled reasons for pursuing PAp goals could help clarify when PAp goals will be beneficial and when they will be detrimental for learning and achievement.

Notably, although Elliot (2005) argued that achievement goals are by definition rooted in definitions of competence, he also suggested that the reasons underlying the pursuit of an achievement standard (i.e., task-based, intrapersonal, or interpersonal) do not necessarily make reference to competence-related dynamics. Indeed, Elliot (2005, p. 65) suggested that students could pursue PAp goals because their self-worth is at stake or because they were promised a reward for being among the top 5\% of their class. Similarly, Urdan and Mestas (2006), in their qualitative study, noted that some students focus on outperforming others for appearance-based reasons, which are not related to competence-dynamics. This implies that any kind of reason could potentially motivate students' pursuit of PAp goals.

In the current study, we considered students' autonomous and controlled reasons as conceived within SDT, thereby hypothesizing that these reasons would explain additional variance in the learning outcomes beyond PAp goals per se. Specifically, the pursuit of PAp goals for autonomous reasons would be associated with adaptive outcomes, whereas the pursuit of these goals for controlled reasons would be relate to less adaptive or maladaptive outcomes. When pursued for autonomous reasons, individuals are more likely to find the pursuit of PAp goals stimulating, enjoyable, and challenging and thus be more committed to the goal, which is likely to relate to deep cognitive processing. In contrast, the controlled pursuit of PAp goals is likely to be experienced as stressful, which will distract individuals' attention from the learning 
activity and will relate to a lack of task-absorbed engagement in it.

Reeve and Deci (1996) provided indirect evidence for this hypothesis in a laboratory experiment where participants competed against one another on an inherently enjoyable puzzle-solving activity. Two competitive situations were created, one in which participants were strongly pressured to outperform others and one in which the competitive setting was more informational. Results indicated that directly competing in a pressuring interpersonal climate (i.e., "focus fully on being the winner") led to less subsequent intrinsic motivation than competing in an informational context. As the pursuit of PAp goals essentially requires individuals to compete against one another, the study by Reeve and Deci indirectly showed that the effects of pursuing PAp goals depend on whether the context is informational versus controlling. Similar findings were recently reported by Ciani et al. (2010) who found that a performance classroom structure was no longer negatively related to students' mastery goal orientation when students experienced their teachers as autonomy-supportive, presumably because the performance environment was experienced as informational instead of controlling under these circumstances.

\section{Perfectionism as antecedent \\ of performance-approach goals and regulations of goal pursuits}

An additional important aim of the present research was to examine whether learners' perfectionist orientations would be associated with the pursuit of PAp goals and the regulations governing their PAp goal pursuits. We focused on perfectionism as a potential precursor of PAp goals because a central characteristic of perfectionism involves the setting of high personal standards for performance and achievement (Blatt 1995; Shafran and Mansell 2001). As such, this personality characteristic is highly relevant to the study of performance goals.

It is important to note that recent theorizing does not consider perfectionism to be a unitary construct but instead distinguishes between adaptive or normal and maladaptive or neurotic perfectionism (Dunkley et al. 2006; Enns et al. 2002; Frost et al. 1993; Hamachek 1978). Adaptive perfectionism refers to the setting of high standards, and studies have found that, after removing the variance shared with maladaptive perfectionism, adaptive perfectionism was related to positive outcomes such as self-esteem, low depression (e.g., Soenens et al. 2005), positive affect when taking exams (Bieling et al. 2003), and higher grades (e.g., Accordino et al. 2000; Bieling et al. 2003; Rice and Slaney 2002).
Although the setting of high standards is not in itself pathological, it may become more maladaptive when it is accompanied by negative self-evaluative tendencies. Maladaptive perfectionists are highly self-critical and typically experience failure as a blow to their self-worth. Because their self-worth is at stake during activity engagement, maladaptive perfectionists tend to continually doubt their actions, to feel anxious about making mistakes, and to ruminate about their mistakes (Frost et al. 1997), which renders them vulnerable to internalizing problems (e.g., Blatt 1995), test anxiety (Mills and Blanstein 2000), negative affect when taking exams (Bieling et al. 2003), feelings of academic incompetence, and lower grades (e.g., Grzegorek et al. 2004).

In line with previous research (Ommundsen et al. 2005), we hypothesized that both types of perfectionism would relate positively to the pursuit of PAp goals, as the setting of high expectations for oneself-the common conceptual feature of both types of perfectionism-is likely to make one more prone to placing value on outperforming others. Moreover, in line with Miquelon et al. (2005), we hypothesized that, whereas adaptive perfectionism would be associated with an autonomous regulation of PAp goals, maladaptive perfectionism would be associated with a controlled regulation of PAp goals. Because adaptive perfectionists would view outperforming others as a challenge and a chance for increased learning, they would pursue PAp goals primarily for autonomous reasons. In contrast, because of the strong self-critical tendencies that characterize maladaptive perfectionism, maladaptive perfectionism is likely to be associated with a pressured pursuit of PAp goals. Finally, in an integrated process model we examined whether the autonomous and controlled pursuit of PAp goals would either fully or partially mediate the relations of both types of perfectionism to the learning outcomes. The role of perfectionism was examined in Study 2.

\section{Present research}

In two studies we examined the association between PAp goals, autonomous and controlled reasons for pursuing PAp goals and a variety of learning outcomes, namely, selfregulated learning (Studies 1 and 2), performance, cheating attitudes and cheating behavior (Study 2). Within the educational literature, self-regulated learning has been generally defined as the generation of thoughts, emotions, and actions that are oriented towards accomplishment of student goals (Schunk and Zimmerman 1994). Although researchers have introduced somewhat different taxonomies of self-regulated learning (e.g., Boekaerts 1997; Schunk and Zimmerman 1994), most of these models 
emphasize the importance of three components: (1) the use of cognitive strategies to process, learn, and understand the study material (e.g., selecting main ideas); (2) meta-cognitive strategies, which refer to the cognitive monitoring and supervision of the learning activity (e.g., time management, concentration); and (3) engagement or will, which reflects the effort students put into the learning activity (e.g., persistence, attitude) (Pintrich and DeGroot 1990). To assess these aspects of self-regulated learning, we made use of several subscales of the Learning and Study Strategy Inventory (LASSI; Weinstein and Palmer 1987). In addition to self-regulated learning, we also included in Study 2 a measure of self-reported cheating and attitude towards cheating (Anderman et al. 1998; Newstead et al. 1996), as we expected these to be differentially predicted by the reasons for learners' PAp goal pursuits.

Our main hypothesis was that the reasons for pursuing PAp goals would explain incremental variance in the outcomes beyond that predicted by the PAp goals per se, with autonomous and controlled reasons being, respectively, positively and negatively related to the various aspects of self-regulated learning and academic achievement. In addition to these hypothesized main effects of reasons for pursuing PAp goals, we explored three other issues. First, we examined whether PAp goals and underlying regulations would account for independent variance in the outcomes once entered simultaneously in the analyses. We did so because it is possible that the initial associations between PAp goals and outcomes would disappear after inserting the reasons for pursuing the goals. Second, we examined whether the associations of regulations of PAp goals with educational outcomes would hold even after taking into account students' MAp goal pursuit. Third, we examined interactions between goals and reasons, which would allow us to determine whether autonomous and controlled reasons for pursuing PAp goals would have similar relations to educational outcomes for individuals low versus high in their endorsement of PAp goals. Perhaps, the two reasons matter differently for individuals strongly endorsing PAp goals versus weakly endorsing PAp goals.

\section{Study 1}

Method

\section{Participants}

Participants were 150 10th to 12th grade students (64\% female) from a secondary school in Flanders (Belgium) who provided informed consent for participation. Students completed questionnaires during a 45-minute class period. At least one researcher was always present during data collections. Participation was voluntary, and anonymity was guaranteed. The study protocol was approved by the ethical committee of the university.

\section{Measures}

Several questionnaires were already available in Dutch, the participants' native language. For questionnaires needing to be translated from English to Dutch, we followed the guidelines of the International Test Commission (Hambleton 1994). Unless otherwise noted, items were scored on 5-point Likert scales, ranging from 1 (strongly disagree) to 5 (strongly agree).

Achievement goals The strengths of participants' PAp and MAp goals were each assessed with three items from the Achievement Goal Questionnaire developed by Elliot and McGregor (2001). The items were slightly adjusted as they pertained to participants' achievement goals for schooling in general rather than being class specific. We measured only the approach orientation of both the performance goals (e.g., "My goal at school is to get a better grade than most other students") and the mastery goals (e.g., "It is important for me to understand the content of the study material as thoroughly as possible") because the aim of this research was to shed further light on the effects of pursing PAp goals controlling for MAp goals. The three items for the subscales were averaged to form PAp goal and MAp goal subscores. Internal consistencies for PAp $(\alpha=.78)$ and MAp goals $(\alpha=.86)$ were satisfactory. Construct and predictive validity of these translated scales was demonstrated by DeCoster et al. (2007).

Autonomous and controlled regulation of PAp goals After participants responded to each of the three PAp goal items we asked why they pursued this goal in order to obtain a goal-specific assessment of the reasons for their PAp goal pursuit. Then, two items were presented that assessed autonomous reasons ("Because I find this a highly stimulating and challenging goal", intrinsic reason; "Because I find this a personally valuable goal", identified reason) and two items assessed controlled reasons ("Because I have to comply with the demands of others such as parents, friends, and teachers", external reason; "Because I would feel bad, guilty, or anxious if I didn't do so", introjected reason). This procedure is identical to the one developed by Sheldon and Kasser (1995) who focused on the reasons behind individuals' life goals rather than their PAp goals. Scale scores for controlled regulation $(\alpha=.83)$ and autonomous regulation $(\alpha=.84)$ were created by averaging the 6 external and introjected regulation items and the 6 identified and intrinsic regulation items, respectively. 
Learning and study strategies Students' awareness about and use of learning and study strategies were assessed by several subscales of a validated Dutch version (De Bilde et al. 2010; Lacante and Lens 2005) of the Learning and Study Strategy Inventory (LASSI; Weinstein and Palmer 1987). The items were rated on a 5-point Likert scale ranging from 1 (Not at all typical of me) to 5 (Very much typical of me), and all referred to schooling in general rather than to the use of learning and study strategies in a particular class. The following subscales of the LASSI were administered: (1) Information Processing, assessing how well students make use of imagery, verbal elaborations, organization strategies, and reasoning skills to process new information, and make use of skills to build bridges between what they already know and what they are trying to learn and remember (e.g., "To help me remember new principles we are learning in class, I practice applying them"; 8 items; $\alpha=.79$ ); (2) Selecting Main Ideas, assessing students' ability to distinguish important information for further study from less important information and supporting details (e.g., "I have difficulty identifying the important points in my reading" (reverse scored); 5 items; $\alpha=$.66); (3) Time Management, assessing students' use of time management strategies for academic tasks (e.g., "I find it hard to stick to a study schedule" (reverse scored); 8 items; $\alpha=.58$ after dropping one item that reduced internal consistency); (4) Concentration, assessing students' ability to direct and maintain their attention on academic tasks (e.g., "My mind wanders a lot when I study" (reversed scored); 8 items; $\alpha=.83$ ); (5) Test Anxiety, assessing the degree to which students worry about school and their academic performance (e.g., "When I am studying, worrying about doing poorly in a course interferes with my concentration," (8 items); $\alpha=.78$; in this study higher scores mean higher levels of test anxiety, which is different from what the manual shows); (6) Attitude, assessing students' interest in college and achieving academic success (e.g., "I do not care about getting a high school education, I just want to get a good job" (reverse scored); 8 items; $\alpha=.67$ ); (7) Persistence, assessing students' diligence, self-discipline, and willingness to exert effort necessary to successfully complete academic requirements (e.g., "When work is difficult I either give up or study only the easy parts" (reverse scored); 8 items; $\alpha=.71)$. Selecting Main Ideas and Information Processing serve as indicators of cognitive processing, Time Management, Concentration, and Test Anxiety serve as indicators of meta-cognitive processing, and Attitude and Persistence serve as indicators of engagement and will (see Wolters 2004 for a similar differentiation).

\section{Results}

\section{Preliminary analyses}

Independent samples $t$-tests indicated that female, relative to male, participants scored significantly higher on MAp goals $[M=3.91, \quad \mathrm{SD}=.88$ vs. $M=3.60, \quad \mathrm{SD}=.84$; $t(148)=-2.08, p<.05]$ and on test anxiety $[M=2.79$, $\mathrm{SD}=.77$ vs. $M=2.46, \quad \mathrm{SD}=.76 ; t \quad(148)=-2.78$, $p<.01]$. No other significant gender effects emerged. Therefore, when predicting test anxiety, we statistically controlled for gender effects. No grade effects were found. Means, standard deviations and correlations between the study variables can be found in Table 1 .

Table 1 Means, standard deviations, and intercorrelations for measured variables—Study 1

\begin{tabular}{|c|c|c|c|c|c|c|c|c|c|c|c|c|}
\hline & Mean & SD & 1 & 2 & 3 & 4 & 5 & 6 & 7 & 8 & 9 & 10 \\
\hline 1. MAp goals & 3.80 & .87 & & & & & & & & & & \\
\hline 2. PAp goals & 2.44 & 1.07 & $.41 * * *$ & - & & & & & & & & \\
\hline $\begin{array}{l}\text { 3. Autonomous regulation of } \\
\text { PAp goals }\end{array}$ & 2.42 & .96 & $.42 * * *$ & $.73 * * *$ & - & & & & & & & \\
\hline $\begin{array}{l}\text { 4. Controlled regulation of PAp } \\
\text { goals }\end{array}$ & 2.04 & .87 & $.26 * * *$ & $.49 * * *$ & $.41 * * *$ & - & & & & & & \\
\hline 5. Information processing & 3.02 & .74 & $.46^{* * * *}$ & $.28 * * *$ & $.33 * * *$ & .15 & - & & & & & \\
\hline 6. Selecting main ideas & 3.37 & .78 & $.29 * * *$ & .12 & $.20 *$ & -.05 & $.42 * * *$ & - & & & & \\
\hline 7. Time management & 2.60 & .73 & $.35 * * *$ & $.28 * * *$ & $.34 * * *$ & .03 & $.34 * * *$ & $.30 * * *$ & - & & & \\
\hline 8. Concentration & 2.99 & .81 & $.23^{* *}$ & .15 & $.25 * *$ & -.11 & $.24 * *$ & $.54 * * *$ & $.59 * * *$ & - & & \\
\hline 9. Test anxiety & 2.66 & .77 & $.25 * *$ & .15 & .08 & $.31 * *$ & .04 & $-.33 * * *$ & -.14 & $-.47 * * *$ & - & \\
\hline 10. Attitude & 3.37 & .65 & $.24 * *$ & .09 & $.19^{*}$ & $-.16^{*}$ & $.23 * *$ & $.45 * * *$ & $.47 * * *$ & $.66 * * *$ & $-.25 * * *$ & - \\
\hline 11. Persistence & 3.08 & .76 & $.39 * *$ & $.30 * * *$ & $.35^{* * *}$ & .03 & $.44 * * *$ & $.36^{* * * *}$ & $.49 * * *$ & $.48 * * *$ & -.08 & $.39 * * *$ \\
\hline
\end{tabular}

$* p<.05 ; * * p<.01 ; * * * p<.001$ 


\section{Primary analyses}

To examine the central questions of this research, we performed a series of hierarchical multiple regression analyses. In Step 1, outcomes were regressed onto PAp goal pursuit, and the autonomous and controlled regulations were added as additional predictors in Step 2 to examine whether these reasons would account for incremental variance in the outcomes. Further, comparison of the change in association between PAp goals and outcomes in Steps 1 and 2 indicates whether PAp goals still yields an independent association with educational outcomes after adding the reasons for pursuing the PAp goals to the regression equation. MAp goals were then added in a third step to examine whether the obtained associations of PAp goals and the regulations with educational outcomes would hold after controlling for the effects of MAp goals. Finally, in Step 4, we entered two-way interactions between each reason for pursuing PAp goals and PAp goal strength, as well as between controlled and autonomous reasons for pursuing PAp goals. Interaction terms were created by multiplying centered means. The results of the first three steps in these multiple regression analyses can be found in Table 2. Step 4 is not reported in the table because only one out of 21 of the two-way interactions was significant.
As can be seen in Step 1, after controlling for gender, PAp goals had positive associations with three out of seven outcomes, namely, information processing, time management, and persistence. Adding autonomous and controlled regulation to pursue PAp goals in Step 2 resulted in a significant increase in explained variance in all outcomes. Specifically, autonomous regulation of PAp goals was significantly positively related to information processing, selecting main ideas, time management, concentration, attitude, and persistence, but was unrelated to test anxiety. Controlled regulation of PAp goals was negatively related to concentration and attitude and positively related to test anxiety. Importantly, the association of PAp that was observed in Step 1 of the regression equation was no longer significant in Step 2 when autonomous and controlled regulations were added. Adding MAp in the regression equation in Step 3 slightly weakened the results for autonomous regulation of PAp goals, but it also strengthened the results for controlled regulation of PAp goals. Specifically, controlled regulation of PAp goals was now significantly negatively related to selecting main ideas, time management, and persistence. Finally, the only interaction in Step 4 that reached significance was between autonomous and controlled regulation of PAp goals on selecting main ideas $(\beta=-.27, p<.001)$. With just one out of 21 interactions being significant, there is no

Table 2 Beta coefficients from hierarchical multiple regression analyses with achievement goals and reasons as predictors for self-regulated learning-Study 1

\begin{tabular}{|c|c|c|c|c|c|c|c|c|c|c|c|c|}
\hline \multirow[t]{2}{*}{ Step } & \multicolumn{3}{|c|}{ Information processing } & \multicolumn{3}{|c|}{ Selecting main ideas } & \multicolumn{3}{|c|}{ Time management } & \multicolumn{3}{|c|}{ Concentration } \\
\hline & 1 & 2 & 3 & 1 & 2 & 3 & 1 & 2 & 3 & 1 & 2 & 3 \\
\hline PAp goals & $.28 * *$ & .09 & .02 & .12 & .01 & -.05 & $.28 * * *$ & .13 & .07 & .15 & .04 & .00 \\
\hline Autonomous regulation of PAp goals & & $.26^{*}$ & .16 & & .26 & .20 & & $.31 * *$ & $.25^{*}$ & & $.32 * *$ & $.28^{*}$ \\
\hline Controlled regulation of PAp goals & & .00 & -.03 & & -.16 & $-.18 *$ & & -.16 & $-.18^{*}$ & & $-.26^{* *}$ & $-.28 * *$ \\
\hline MAp goals & & & $.42 * * *$ & & & $.28 * *$ & & & $.27 * * *$ & & & $.20^{*}$ \\
\hline R-square & .08 & .11 & .24 & .01 & .06 & .12 & .08 & .14 & .20 & .02 & .11 & .14 \\
\hline \multirow[t]{3}{*}{$\mathrm{R}$-square change } & & $.03 *$ & $.13^{* *}$ & & .0 & $.06 * *$ & & $.06 * *$ & $.06^{* *}$ & & $.09 * *$ & $.03 *$ \\
\hline & \multicolumn{4}{|c|}{ Test anxiety } & \multicolumn{3}{|c|}{ Attitude } & \multicolumn{4}{|c|}{ Persistence } & \\
\hline & 1 & 2 & 3 & & 1 & 2 & 3 & 1 & 2 & 3 & & \\
\hline PAp goals & .15 & .07 & .03 & & .09 & .01 & -.04 & $.29 * * *$ & .14 & .0 & 8 & \\
\hline Autonomous regulation of PAp goals & & -.12 & -.16 & & & $.30 *$ & $.24 *$ & & $.31 * *$ & & 24 & \\
\hline Controlled regulation of PAp goals & & $.34 * * *$ & $.32 *$ & & & $-.29 * *$ & $-.30 * * *$ & & -.16 & -.1 & $9 *$ & \\
\hline MAp goals & & & $.18^{*}$ & & & & $.23 * *$ & & & & $1 * * *$ & \\
\hline $\mathrm{R}$-square & .06 & .15 & .18 & & .01 & .10 & .15 & .09 & .15 & .2 & 2 & \\
\hline $\mathrm{R}$-square change & & $.09 * *$ & $.03 *$ & & & $.09 * *$ & $.05^{* *}$ & & $.06^{* *}$ & & $77 * *$ & \\
\hline
\end{tabular}

$* p<.05 ; * * p<.01 ; * * * p<.001$ 
indication that this is a meaningful finding. In the final step of the regression analyses, between 12 and $24 \%$ of the variance in the outcomes were explained. ${ }^{3,4}$

\section{Brief discussion}

This study revealed several important findings. First, the results for PAp goals generally mirror the findings obtained in previous work within achievement goal theory, as PAp goals were found to be positively related to three out of seven outcomes. Second, when the autonomous and controlled reasons for pursuing PAp goals were entered, one or the other or both reasons predicted significant variance over and above that predicted by PAp goals in all outcomes, thus providing initial evidence for our main hypothesis. Specifically, autonomous regulation of PAp goals was positively associated with (1) cognitive processing, as indexed by information processing and selecting main ideas, (2) meta-cognitive processing, as indexed by higher time management and higher concentration, and (3) engagement, as indexed by a more positive attitude and more persistence; whereas controlled regulation of PAp goals was negatively related to concentration and to positive attitude and was positively related to test anxiety. Third, when the strength of PAp goals and the reasons for pursuing them competed for variance, the associations of the PAp goals with outcomes dropped below significance in each of the cases where PAp goals had been significant. This suggests that students' reasons for pursuing PAp goals are more strongly related to their learning experiences than are the pursuit of PAp goals themselves. Fourth, the independent associations of individuals' autonomous and controlled reasons for pursuing PAp goals remained largely

\footnotetext{
3 As some students scored low on PAp goals it might have seemed odd for them to answer why they pursued these goals, so we repeated the full sequence of analyses after removing those students with an average PAp-goal score below 2. The relations of autonomous and controlled regulation of PAp goals to outcomes in both subsamples $(N=103$ for Study $1 ; 69 \% ; N=158$ for Study $2 ; 80 \%)$ of high PApgoal oriented students were essentially identical to the results obtained in the total sample.

${ }^{4}$ To detect multicollinearity, we examined its impact on the precision of estimation of the regressors, which is reflected in the Variance Inflation Index (VIF; Fox 1991). When entering PAp goals and reasons in the second step, we found that no single VIF exceeds the cut-off criterion of 4 (maximum VIF $=2.41$ and 2.06 in Study 1 and 2 , respectively). Similarly, the collinearity diagnostics table, which represents an alternative method of assessing the problem of multicollinearity, yielded no condition indices over 15 (Hair et al. 2006); maximum condition index $=12.13$ and 12.54 in Study 1 and 2 , respectively). These observations allowed us to conclude that there is no serious degrading in the precision of estimation of parameters (Miles and Shevlin 2001) and that the main effects of PAp goals and regulations can be interpreted in a reliable manner.
}

significant after entering MAp in the regression equation, and MAp goals did contribute additional predictive value in most outcomes. Fifth, the lack of interactions between reasons for PAp goal pursuits and strength of PAp goals indicates that the observed relations between autonomous and controlled reasons for pursuing PAp goals and learning outcomes apply to all individuals. Thus, even students with weak endorsement of PAp goals will display more optimal learning when autonomously regulating their weakly held PAp goals.

\section{Study 2}

Study 2 had three goals. First, we aimed to replicate the findings regarding the differential associations of autonomous and controlled regulations of PAp goals with educational outcomes. Second, we extended the number of outcomes by including assessments of cheating and cheating attitudes as well as objectively assessed achievement. We expected individuals who autonomously pursue PAp goals to be less prone to cheating and to display a more negative attitude toward cheating, whereas those who pursue PAp goals for controlled reasons would be more prone to cheating and to displaying a more approving attitude towards cheating. It seemed that cheating would be viewed as an appropriate way to beat others when students felt a strong pressure to do so. In addition, we examined whether a controlled regulation of PAp goals would negatively relate to achievement, whereas an autonomous regulation of PAp goals would positively relate to it. Third, we examined whether learners' adaptive and maladaptive perfectionism would relate to their pursuit of PAp goals and the regulation of PAp goal pursuits. We expected that both types of perfectionism would be associated independently with the pursuit of PAp goals as the desire to outperform others would reflect a direct expression of one' setting of high personal standards, which is central to both types of perfectionism. However, we expected the types of perfectionism to relate differently to the two reasons for pursuing PAp goals. Because the tendency to outperform others would be perceived as a interesting challenge for adaptive perfectionists and as a pressuring threat for maladaptive perfectionists, adaptive and maladaptive perfectionism were expected to relate, respectively, to autonomous and controlled regulation of PAp goals. Finally, we tested an integrated model in which the regulations behind PAp goals were hypothesized to at least partially mediate the associations between both types of perfectionism and outcomes, whereby the learning correlates of adaptive and maladaptive perfectionism would be specifically mediated, respectively, by autonomous and controlled regulation of PAp goals. 


\section{Method}

\section{Participants and procedure}

One hundred and ninety 11 th and 12 th grade students (46\% female) from a secondary school in Flanders (Belgium) participated in the research and provided informed consent for their participation. We followed the same procedure as in Study 1. Students completed the questionnaires during a 45-minute class period 2 weeks before the Christmas exams. At least one of the researchers was always present during data collection. Participation was voluntary, and anonymity was guaranteed. The study protocol was approved by the ethical committee of the university.

\section{Measures}

Perfectionism Participants completed three scales from the Multidimensional Perfectionism Scale (MPS; Frost et al. 1990), namely the Concern over Mistakes scale (9 items, e.g., "People will probably think less of me if I make a mistake"), the Doubts about Actions scale (4 items, e.g., "Even when I do something very carefully, I often feel that it is not quite right"), and the Personal Standards scale (7 items, e.g., "I set higher goals for myself than most people"). Past factor-analytical studies have shown that items from the Concern over Mistakes and the Doubts about Actions scales load on a single factor, which has been labeled 'maladaptive perfectionism' or 'evaluativeconcerns perfectionism', whereas the items of the Personal Standards scale load on a separate factor labeled 'adaptive perfectionism' or 'positive-striving perfectionism' (e.g., Dunkley et al. 2006; see Soenens et al. 2005 for a validated Dutch version of this scale). The items assessing Concern over Mistakes and Doubts about Actions were averaged to form an index of maladaptive perfectionism, whereas the items assessing Personal Standards were averaged to form an index of more adaptive perfectionism. Cronbach's alpha was .86 and .81 for maladaptive perfectionism and adaptive perfectionism, respectively.

Achievement goals The strength of participants' achievement goal striving was assessed in the same way as in Study 1 . The items were averaged to create PAp-goal and MAp-goal scores. Internal consistencies for PAp goals $(\alpha=.84)$ and MAp goals $(\alpha=.86)$ were good.

Autonomous and controlled regulation of PAp goals Participants' autonomous reasons for pursuing PAp goals were assessed in the same way as in Study 1. Participants' controlled reasons for pursuing PAp goals were assessed in a more extensive way by including controlled regulation items that were worded with both approach terms (i.e., "because others such as parents, friends, teachers etc. will only reward me if I pursue this goal;" "because I will feel more proud of myself if I pursue this goal") and avoidance terms (i.e., "because others such as parents, friends, teachers etc. would get mad at me if I didn't pursue this goal;" " because I would feel guilty, ashamed or anxious if I didn't pursue this goal"). The inclusion of approach oriented items seemed important as controlled reasons can involve either avoiding negative outcomes or approaching positive ones (Assor et al. 2009). We created a composite autonomous-regulation score by averaging the two intrinsic and two identified regulation items $(\alpha=.90)$ and a composite controlled-regulation score by averaging the four external and introjected regulation items $(\alpha=.90)$.

Learning and study strategies The same learning and study strategies as in Study 1 were assessed. Internal consistencies of information processing $(\alpha=.78)$, selecting main ideas $(\alpha=.75)$, time management $(\alpha=.64)$, concentration $(\alpha=.87)$, text anxiety $(\alpha=.86)$, attitude $(\alpha=.63)$, and persistence $(\alpha=.77)$ were satisfactory.

Achievement We obtained the exam results from the official school records for the December exam series that took place two weeks after students' participation in the study. This measure of objective and prospective performance was calculated by averaging the students' scores for each of the 13-15 courses in which they took exams.

Cheating The cheating measures were taken from Anderman et al. (1998). Two different aspects of cheating were assessed: (a) the extent to which students were effectively cheating during tests (5 items; e.g., "I copy answers from other students on tests"; $\alpha=.85$ ), which were answered on a 5-point Likert scale varying between 1 (Completely not true) to 5 (Completely true); (b) their beliefs about the acceptability of cheating, which was assessed with the items: "How serious do you think it is if somebody cheats during tests?", "If you were sure you wouldn't get caught, would you cheat during tests?" and "It is okay to cheat during tests." Internal consistency (Cronbach's alpha) for this three-item scale was .70.

\section{Results}

\section{Preliminary analyses}

An independent samples $t$-test indicated that female relative to male participants scored lower on controlled regulation of PAp goals $(M=2.24$ vs. $M=2.51 ; t(188)=-2.47$, $p<.05)$ and higher on MAp goals $(M=3.94$ vs. $M=3.65 ; t(188)=2.58, p<.01)$. As for the outcome variables, female relative to male participants scored higher 
on concentration $(M=3.16$ vs. $M=2.93, t(188)=2.07$, $p<.05)$, test anxiety $(M=3.03 \quad$ vs. $M=2.74$; $t(188)=2.58, p<.01)$, persistence $(M=3.32$ vs. $M=$ $3.01 ; t(188)=3.51, p<.001)$, and lower on a positive attitude towards cheating $(M=2.88$ vs. $M=3.18$; $t(188)=-2.47, p<.05)$, self-reported cheating $(M=$ 2.28 vs. $M=2.55 ; t(188)=-1.98, p<.05)$, and higher on objective performance $(M=66.20$ vs. $M=63.38$; $t(188)=2.91, p<.01)$. Therefore, we controlled for gender effects in all primary analyses. No grade effects were found. The means, standard deviations, and correlations among study variables can be found in Table 3 .

\section{Primary analyses}

PAp goals and regulations As in Study 1, we conducted a series of hierarchical regressions in which outcomes were regressed onto PAp goals in Step 1, the autonomous and controlled regulation of PAp goals in Step 2, MAp goals in Step 3, and two-way interactions between reasons for pursuing PAp goals and PAp goal strength in Step 4. Results can be found in Table 4. After controlling for gender, PAp goals were found to be positively related to information processing, concentration, test anxiety and persistence, while being negatively related to cheating attitude. The inclusion of autonomous and controlled regulation in Step 2 resulted in a significant increase in explained variance in all outcomes. Notably, and similar to Study 1, most of the significant associations between PAp goals and outcomes dropped below significance when the reasons were added. The only remaining findings for PAp goals were the positive relation with test anxiety and the negative association with cheating attitude. As for the associations of the regulations for PAp goals, autonomous and controlled regulation showed significant independent yet opposite associations with selecting main ideas, time management, concentration, test anxiety, attitude, persistence, cheating attitude and cheating behavior. Autonomous regulation was additionally positively related to information processing, whereas controlled regulation was negatively related to objective performance. These results were slightly weakened by entering MAp goals in the regression equation, with the relations of autonomous and controlled regulation, respectively, to time management and cheating behavior becoming non-significant. Finally, and similar to Study 1, only one out of 30 interactions was found to be significant: autonomous reasons underlying PAp goals interacted with PAp goals in the prediction of information processing. With only 1 of 30 significant twoway interactions being significant, and especially because it was not the same one that was significant in Study 1, there is no evidence for any moderation of PAp goal relations to outcomes by the autonomous and controlled reasons for the goal pursuits. Overall, the set of predictors explained between 10 and $33 \%$ of the variance in outcomes. 5

Structural equation modeling To examine whether PAp goals and regulatory reasons for the goal pursuits would account for the association between perfectionism and learning outcomes, we tested a series of structural equation models. In doing so, we followed the procedures outlined by Holmbeck (1997). First, we examined whether each type of perfectionism would yield relations with the learning outcomes and would relate to the hypothesized mediating variables (i.e., PAp goals and reasons); second, we tested a full mediation model in which the two types of perfectionism would only be indirectly related to the learning outcomes through the hypothesized mediators; third, we tested a partial mediation model which included both direct paths from both types of perfectionism to the learning outcomes and indirect paths through students' PAp goals and their underlying reasons. Full mediation is demonstrated when the addition of direct paths does not improve model fit compared to the model that does not include such direct paths.

Data screening using Prelis 2.54 (Joreskog and Sorbom 1996) indicated partial non-normality of the data, both at the univariate and the multivariate level. Therefore, in addition to the covariance matrix, in all subsequent models we also used the matrix of asymptotic covariances as input, and we inspected the Satorra-Bentler Scaled chi-square (SBS- $\chi^{2}$, Satorra and Bentler 1994) to correct for this nonnormality. Solutions were generated on the basis of maximum-likelihood estimation. To assess the fit of the model to the observed data, we employed the Satorra-Bentler Scaled chi-square statistic, the comparative fit index (CFI; Bentler 1990), the root mean square residual (SRMR; Steiger 1990), and the Root Mean Square Error of Approximation (RMSEA). A non-significant chi-square indicates a well fitting model (Bollen 1989); fit indices above .90 are considered acceptable, as is an SRMR below

\footnotetext{
$\overline{5}$ To examine whether the observed different associations between autonomous and controlled reasons underlying of PAp goals and outcomes were due to the specific reasons for pursuing the PAp goals rather than being a reflection of positive relations of autonomous and controlled functioning with outcomes at the personality level (Deci and Ryan 1985), we repeated the full sequence of regression analyses while entering students' general, trait-like orientation towards autonomous and controlled functioning as covariates. All of the observed associations between the autonomous and controlled regulation of PAp goals and learning outcomes remained significant, suggesting that the observed associations are goal-specific and cannot be accounted for by underlying personality traits towards autonomy or control.
} 


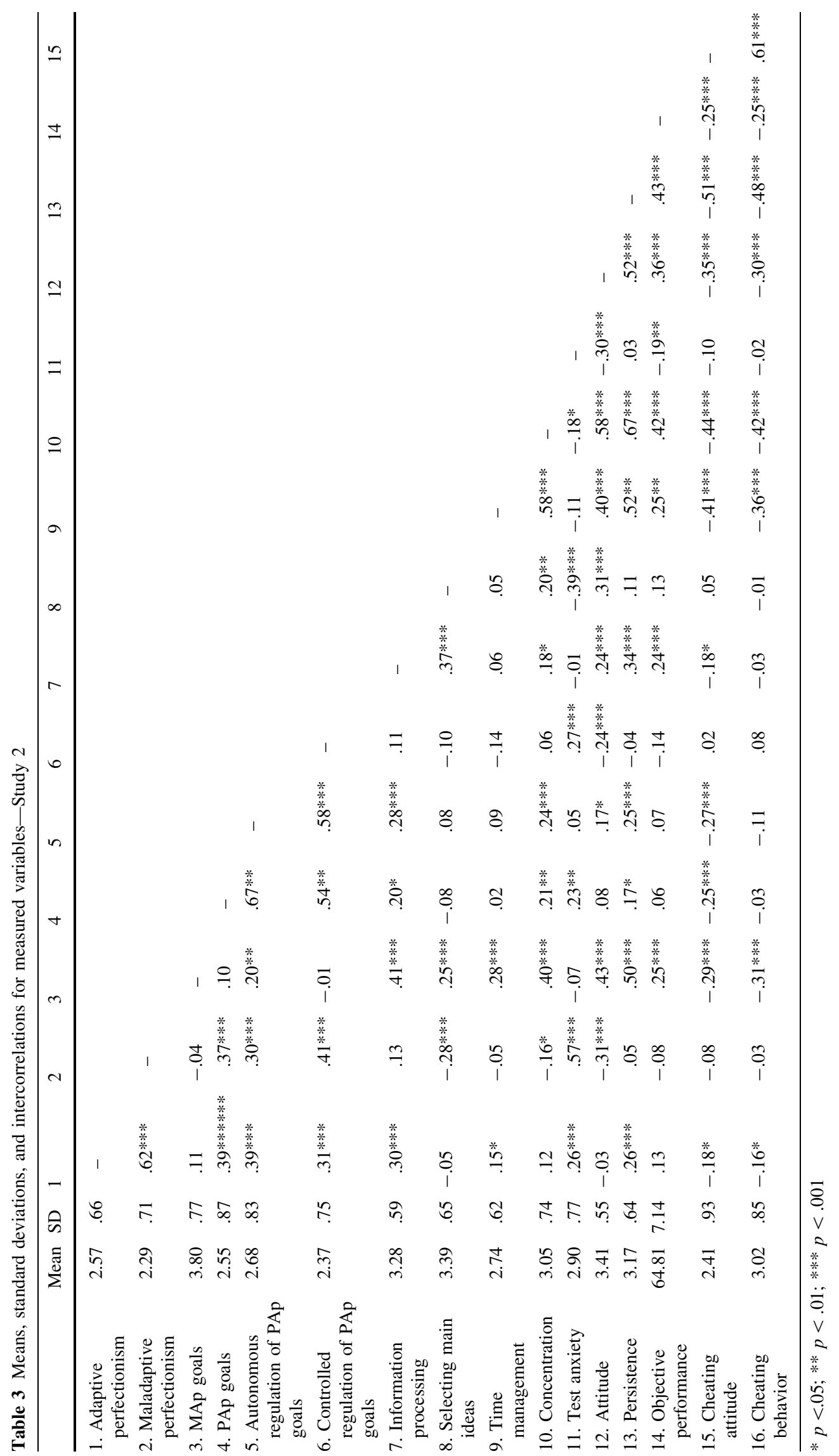




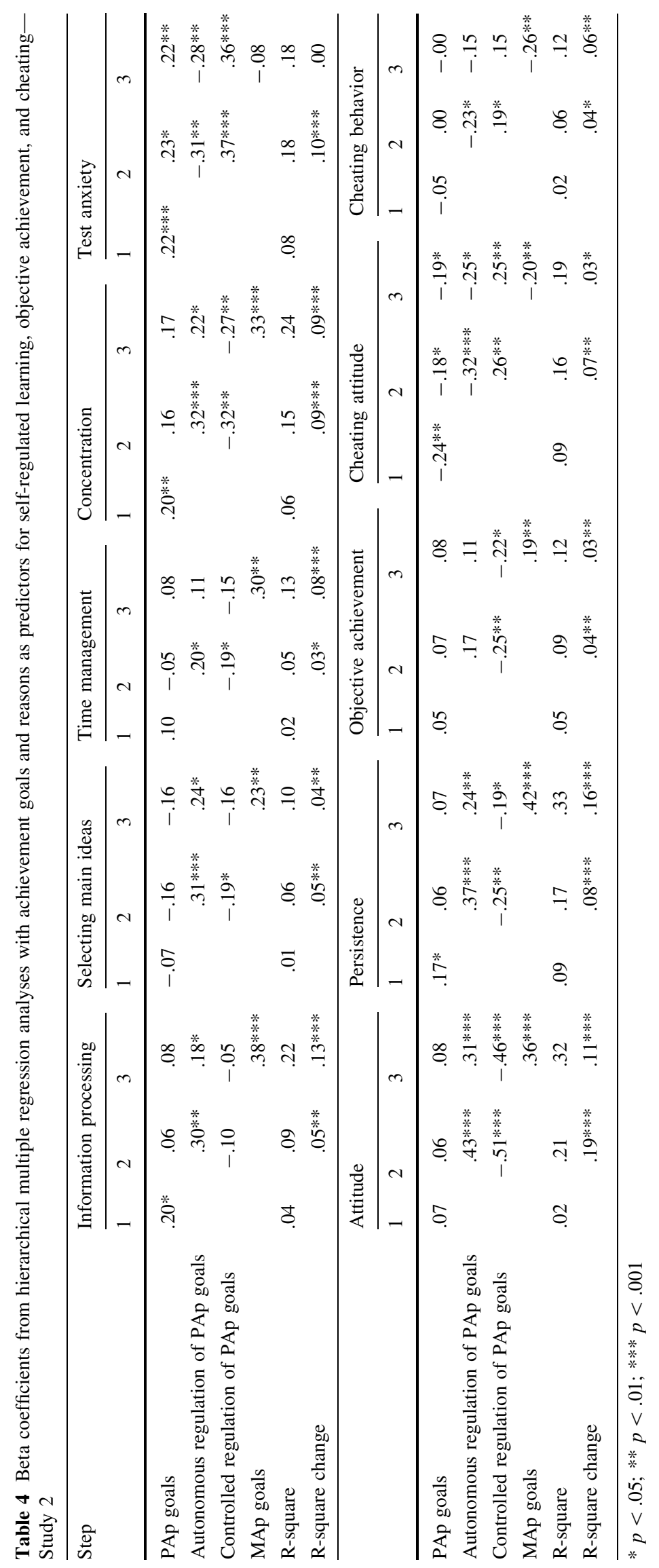


.08 (Browne and Cudeck 1993) and a RMSEA below .08 (Hu and Bentler 1999).

To reduce the number of learning outcomes to be estimated in the path model, we created four different composite scores by z-scoring and averaging the subcomponents that define each composite score, that is, (1) a cheating composite was based upon the cheating attitude and cheating behavior subscales, (2) a cognitive processing composite was based upon the subscales information processing and selecting main ideas, (3) a meta-cognitive composite was based upon the subscales time management, concentration, and test anxiety (reverse scored), and (4) an engagement composite was based upon the subscales attitude and persistence. In addition to these composite scores, we also used objective performance as a fifth critical learning outcome. Finally, in light of the gender differences on various outcomes, we controlled for gender effects in all of the models.

We began by testing the association between perfectionism and learning outcomes. Given that we allowed a path from both types of perfectionism and gender to all four learning outcomes, the model was fully saturated and, hence, yielded a perfect fit. Specifically, both types of perfectionism yielded significant relations to most outcomes, albeit in opposite directions. Adaptive perfectionism was positively related to cognitive processing $(\beta=.33$, $p<.01)$, meta-cognitive self-regulation $(\beta=.39$, $p<.01)$, determination $(\beta=.43, p<.01)$ and objective achievement $(\beta=.34, p<.01)$, whereas it was negatively related to the cheating composite $(\beta=-.30, p<.01)$. In contrast, maladaptive perfectionism was negatively related to cognitive processing $(\beta=-.29, p<.01)$, meta-cognitive self-regulation $(\beta=-.55, p<.01)$, determination $(\beta=-.39, p<.01)$, and objective achievement $(\beta=-.26$, $p<.01)$, but was unrelated to the cheating composite $(\beta=.11, n s)$.

Next, we examined the links from the two types of perfectionism to PAp goal strength and the two reasons for pursuing the PAp goals. In line with our hypotheses, a path between both types of perfectionism and PAp goals was drawn, whereas only one path between a type of perfectionism to a reason for pursuing PAp goals was allowed, with adaptive perfectionism being related to autonomous reasons and maladaptive perfectionism being related to controlled reasons. This model yielded the following fit: SBS- $\chi^{2}(2, N=190)=2.41 ;$ CFI $=.99 ;$ SRMR $=.03$; $\mathrm{RMSEA}=.05$. As predicted, both adaptive and maladaptive perfectionism were related to PAp goals $(\beta=.29$ and .15 , $p$ 's $<.01$ and 05 , respectively). Further, adaptive perfectionism was positively associated with autonomous reasons underlying PAp goals $(\beta=.37, p<.01)$, whereas maladaptive perfectionism was positively associated with controlled reasons underlying PAp goals $(\beta=.35$, $p<.01)$. Adding the two remaining paths from both types of perfectionism to reasons did not result in a significant improvement in model fit.

Then, we tested a full mediational model to examine whether the associations between both types of perfectionism and learning outcomes could be accounted for by PAp goals and reasons for pursuing the goals. This model yielded the following fit: SBS- $\chi^{2}(12, N=190)=40.10$; $\mathrm{CFI}=.97 ;$ SRMR $=.049 ;$ RMSEA $=.11$. To examine whether the associations between perfectionism and the learning outcomes was fully or partially mediated by PAp goals and underlying reasons, we tested several additional models in which a direct path from one or both types of perfectionism to one single outcome was allowed. The model in which a direct path was drawn from maladaptive perfectionism to meta-cognitive self-regulation yielded a better fit compared to the full mediational model, SBS- $\chi_{\text {diff }}^{2}(1, N=190)=14.10, p<.001$, indicating that the association between maladaptive perfectionism and meta-cognitive self-regulation could only be partially accounted for by PAp and the reasons. The fit of this final model was as follows: $\operatorname{SBS}-\chi^{2}(11, N=190)=26.68$; $\mathrm{CFI}=.98 ; \mathrm{SRMR}=.042 ; \mathrm{RMSEA}=.087$. All the indirect effects of adaptive and maladaptive perfectionism to the learning outcomes were significant, that is, achievement $(z=-1.93, p<.07$ for maladaptive perfectionism), cheating $(z=-3.19, p<.01 ; z=2.35, p<.05)$, cognitive processing $(z=2.64, p<.01 ; z=-2.10, p<.05)$, engagement $(z=-4.00, p<.001 ; z=3.76, p<.001)$, and meta-cognitive processing $(z=3.39, p<.001 ; z=$ $-3.12, p<.001)$. The final model is graphically depicted in Fig. 1. As can be noticed, only the reasons underlying the pursuit of PAp goals served as mediating variables, because PAp goals did not yield any unique association with any of the outcomes in this final model. ${ }^{6}$

\section{Brief discussion}

Study 2 largely replicated the findings of Study 1 while simultaneously extending them. First, as in Study 1, PAp goals showed some positive associations with learning outcomes. Second, also as in Study 1, the reasons underlying the pursuit of PAp goals were related to optimal learning above and beyond the pursuit of PAp goals. Specifically, autonomous regulation of PAp goals was

\footnotetext{
${ }^{6}$ Given that the association between autonomous reasons for PAp goals and objective achievement almost reached significance in the final model $(\beta=.17)$, we performed an additional analysis in which we removed PAp goals. Autonomous reasons were found to yield a significant positive association in this model $(\beta=.20, p<.05)$. This finding contrasts with the observation that PAp goals did not yield a significant association with objective achievement $(\beta=.14, n s)$ after removing autonomous reasons from the model.
} 
Fig. 1 Final mediational model depicting relations between perfectionism and learning outcomes as mediated by performance-approach goals and underlying autonomous and controlled reasons (Study 2). Gender is left out for clarity reasons. $* p<.05 ; * * p<.01$

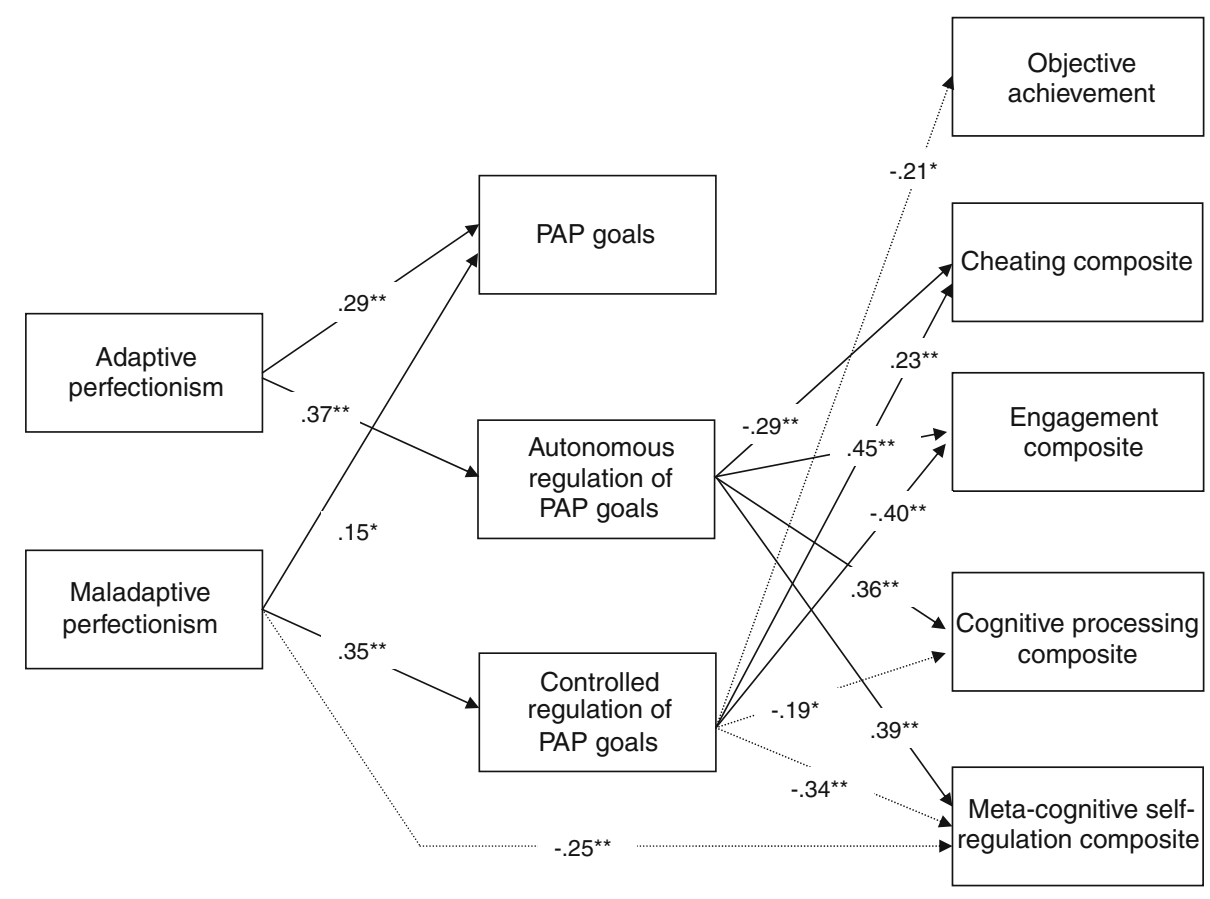

associated with more cognitive processing, better metacognitive self-regulation, more engagement, and less cheating, but it was unrelated to performance. Controlled regulation of PAp goals generally showed the opposite pattern of findings, although controlled regulation was unrelated to information processing but was related negatively to objective performance. Most of these observed relations remained significant after inserting mastery goals as an additional predictor and the observed associations between autonomous and controlled reasons underlying PAp goals applied for individuals scoring both high and low on PAp goals-that is, the goals and reasons did not interact. Third, the initial associations between PAp goals and outcomes largely disappeared after entering autonomous and controlled regulations for PAp goals in the equation, just as had been the case in Study 1. The only exceptions to this pattern were that the positive associations between PAp goals and both test anxiety and cheating attitude remained significant. Fourth, structural equation modeling indicated that adaptive and maladaptive perfectionism served as important individual difference variables being related to learners' pursuit of PAp goals, while the two types yielded different relations to the reasons for pursuing PAp goals: adaptive perfectionists pursued PAp goals for autonomous reasons and maladaptive perfectionists pursued PAp goals for controlled reasons. Fifth, mediational analyses indicated that autonomous and controlled regulation of PAp goals played a significant mediating role in the relations between both types of perfectionism and almost all learning outcomes.

\section{General discussion}

One of the most researched topics within achievement goal theory is the pursuit of a performance-approach (PAp) goal, which is defined as the aim to outperform others on schoolwork and tests (Elliot 1999). This achievement goal has received considerable empirical (e.g., Urdan and Mestas 2006; Darnon et al. 2009) and theoretical (e.g., Brophy 2005) attention, especially since the new millennium, because of its distinct pattern of outcomes: it relates to several positive outcomes (e.g., achievement), some negative outcomes (e.g., test anxiety) and is unrelated to a number of other educational outcomes (e.g., deep level learning). Moreover, some of the observed associations have not been consistently replicated across studies. These findings have led some researchers to argue against promoting this goal (e.g., Midgley et al. 2001), whereas others claim its pursuit might yield some benefits (e.g., Harackiewicz et al. 2002), especially when the learning task only requires rote processing or short-term retention or when the achievement setting is highly competitive and grade-focused. The present research aimed to shed light on these issues by examining whether the autonomous or volitional and the controlled or pressured reasons for pursuing PAp goals would account for additional variance in learning outcomes and would help clarify when the pursuit of PAp goals is adaptive versus harmful. The present research revealed several interesting findings.

First, mirroring previous work, PAp goals were found to be associated with some positive outcomes, such as 
information processing (Studies 1 and 2), time management (Study 1), concentration (Study 2), persistence (Studies 1 and 2), but not with other positives outcomes such as selecting main ideas, a positive scholastic attitude, and objective performance. Further, the strength of PAp goals was positively related to the negative outcome of test anxiety (Study 2). Most of these associations disappeared, however, after entering the autonomous and controlled reasons for pursuing these goals. Thus, when pursuit of interpersonal standards and the reasons behind the standards were separated empirically, as suggested by Elliot (2005), the reasons were more strongly independently related to the learning outcomes than were the goals themselves. Across the two studies, eight of the seventeen relations between PAp goals and outcomes were initially significant, but 6 of those 8 were no longer significant after students' reasons for pursuing PAp goals were entered into the equation. The two exceptions were test anxiety and cheating attitude, both in Study 2. Test anxiety was also included in Study 1 but the relation was not initially significant in that study, and cheating attitude was not included in Study 1.

One might initially think that the finding of autonomous and controlled reasons for pursuing PAp goals, which differentially and more strongly predict outcomes relative to the PAp goals themselves is an indicator of a moderation effect. Moderation of PAp goals by reasons for pursuing them would indicate either that (1) when people are autonomously motivated there would be positive relations between goal strength and outcomes and when they are controlled there would be negative relations, or (2) when people are high in goal strength the autonomous and controlled reasons would relate differently to the outcomes than when the people are low in goal strength However, there was no indication of moderation, as the two-way interactions were not significant. Further, the actual findings that the autonomous and controlled reasons for pursuing the PAp goals predicted outcomes in opposite directions and accounted for more variance in the outcomes than did the goals themselves does not imply that the goals and reasons should interact.

Additionally, one might think that the reduction of nearly all the relations between PAp goals and outcomes to nonsignificance after inserting reasons indicates mediation of the relations between PAp goals and outcomes by reasons. This is possible; however, because these two studies were cross-sectional and correlation-based rather than being longitudinal or experimental, there is no basis for suggesting that PAp goals lead to reasons which then mediate the relation between PAp goals and outcomes. A more straightforward account is simply that the PAp goals are positively correlated with both autonomous and controlled reasons, so they share considerable variance but the reasons have additional unique relations to the outcomes whereas the PAp goals do not.

A second and related finding, which supported the primary hypothesis of this research, was that pursuing PAp goals for autonomous reasons and controlled reasons would be differentially associated with learning outcomes and would thus help explain when the pursuit of PAp goals would be beneficial and when harmful. Autonomous PAp pursuit involves trying to outperform others because one finds the goal challenging and personally important, and controlled PAp pursuit involves trying to outperform others to meet external pressures, such as expectations and rewards, or internal pressures such as self-worth concerns. We predicted that autonomous and controlled regulations of PAp goals would relate differentially to optimal learning, because the psychological meaning of the PAp goals would be different for students who pursue them for different reasons.

In the case of controlled regulation, learners perceive outperforming others as a pressured endeavor so it is likely to disrupt aspects of their learning. In this respect, the controlled regulation of PAp goals was associated with a less effective planning of study time, a more superficial processing of the learning material, and a less concentrated and task-focused engagement in the learning. Further, the controlled regulation of PAp goals was associated with more anxiety when taking tests, presumably because learners' self-worth is more at stake when taking tests. Finally, the controlled regulation of PAp goals was related to a negative attitude towards school in general and to a greater probability to give up in the face of failure.

The pattern of findings for autonomous regulation of PAp goals was strikingly different. When outperforming others is perceived as an exciting challenge and an opportunity for growth, it is associated with a more positive orientation towards school, presumably because an autonomous regulation of PAp goals goes alogn with greater satisfaction of the basic psychological needs for autonomy, competence, and relatedness at school. An autonomous regulation of PAp goals was also associated with a more effective organization of one's study time, a deeper processing of the learning material, and a stronger focus on the learning activity during one's studying. Further, an autonomous regulation was related to greater persistence in the face of difficulties. Most of these findings were replicated across the two studies, although the negative association between autonomous regulation of PAp goals and test anxiety only emerged in Study 2 .

Interestingly, the autonomous and controlled reasons underlying PAp goal pursuits were not only differentially associated with self-regulated learning but were also differentially related to cheating attitudes and behaviors. Previous work within achievement goal theory has shown 
that students adopting a mastery approach were less likely to cheat, whereas students focused on beating others were more likely to cheat (e.g., Anderman et al. 1998; Jordan 2001). The present research replicated the negative correlation of MAp to cheating and further showed that the reasons for pursuing PAp goals were related to students' approval of cheating. Specifically, the degree to which students' pursuit of PAp goals was controlled related positively to their having a positive attitude towards cheating, whereas the degree to which students' pursuit of PAp goals was autonomous related negatively to a positive attitude about cheating. Although the relations to cheating behaviors were weaker and deserve replication, these parallel findings suggest that the two types of reasons are also differentially linked to cheating behaviors.

Some studies have shown that the pursuit of PAp goals was predictive of performance (e.g., Harackiewicz et al. 1997), although others have not confirmed this (e.g., Lau and Nie 2008). In the present research, PAp goals and autonomous regulation of PAp goals were not related to objectively assessed performance, but controlled regulation of PAp goals was negatively related to this outcome, suggesting that feeling pressured to outperform others interferes with performing well on those tests. It remains to be investigated whether these findings can be generalized to other, possibly more competitive, settings, as the selective goal hypothesis would predict that feeling compelled to outperform others might even be beneficial if the interpersonal environment emphasizes interpersonal competition (e.g., Barron and Harackiewicz 2001; Murayama and Elliot 2009). From the perspective of SDT, however, the feeling of being coerced into PAp goal pursuit is unlikely to facilitate performance, especially when the learning requires deep processing and creativity.

A third set of findings concerns perfectionism as an individual-difference predictor of PAp goals and their underlying reasons. Although Elliot and colleagues (e.g., Elliot and McGregor 1999) have examined achievement motivation and fear of failure as personality precursors of achievement goals, the present study is one of the first we know of to focus on perfectionism as an antecedent of PAp goals (see also Stoeber et al. 2009) and underlying reasons. Consistent with the perfectionism literature, both adaptive and maladaptive perfectionism were considered. Whereas both involve setting high achievement standards, the latter also includes a critical evaluation of oneself when one fails to attain the self-imposed standards.

Interestingly, results of structural equation modeling indicated that both types of perfectionism were associated with a stronger endorsement of PAp goals, indicating that pursuit of normative competence results from perfectionists' high standards. Yet, the pursuit of PAp goals could not account for the association between both types of perfectionism and learning outcomes; hence, if we had limited ourselves to the assessment of just PAp goal strength, we would have been unable to explain the effects of perfectionism on the educational outcomes. Due to the assessment of reasons for pursuing PAp goals we were able to gain insight into the explanatory processes linking perfectionism and outcomes. Specifically, the two types of perfectionism were associated with different reasons for pursuing PAp goals. Adaptive perfectionism was associated positively with autonomous regulation of PAp goals, presumably because trying to outperform others is considered highly stimulating and challenging for adaptive perfectionists. Moreover, autonomous regulation of PAp goals fully mediated the positive associations between adaptive perfectionism and learning outcomes. When people set high standards in a flexible manner, they displayed better cognitive and meta-cognitive processing and more engagement and they were more critical of cheating because they pursued the goal of outperforming others volitionally. In contrast, when individuals coupled the pursuit of high standards with critical self-evaluations, they felt coerced to pursue normative competence and were likely to experience the interpersonal competition as threatening, which helps explain why maladaptive perfectionists were more vulnerable to suboptimal learning. Indeed, mediation analyses indicated that the controlled regulation of PAp goals associated with maladaptive perfectionism can fully explain most of the detrimental learning effects related to maladaptive perfectionism. The relation with meta-cognitive strategy use could only be partially accounted for by a controlled regulation underlying PAp goals. One potential reason for this remaining association is that maladaptive perfectionism, operationalized by the subscales of concern over mistakes and doubt about actions, and meta-cognitive self-regulation, with test anxiety as one of its subcomponents, share some item overlap, as the experience of anxiety is part of both constructs.

\section{Limitations and future research}

In spite of its strengths, the present research contains limitations that might be addressed in future studies. An obvious limitation involves the cross-sectional, correlational design that precludes causal inferences. For instance, test anxiety might not only be the result of feeling pressured to outperform others, but experiencing anxiety about tests might also lead students to pursue PAp goals to prove their self-worth. Similarly, maladaptive perfectionism might not only predict controlled functioning, but controlled functioning might also give rise to increased maladaptive perfectionism over time. Second, because the samples were quite homogeneous, future research is 
needed to examine the generalizability of the current findings across samples (e.g., different age groups and SES), across type of academic settings (e.g., highly competitive vs. non-competitive settings), and with respect to other outcomes (e.g., well-being, coping with failure, etc.). Third, most of the variables, except for performance in Study 2, were self-reported, such that shared method-variance might account for some of the observed results. Future research could include behavioral assessments of outcomes, such as persistence and cheating behavior. Fourth, some of the scales that tap into aspects of selfregulated learning had a rather low reliability. Thus, future research would need to confirm the current findings using different questionnaires to measure self-regulated learning.

Experimental research would also be useful as a complement to the present correlational research (e.g., Spray et al. 2006). It would for instance be interesting to explore whether experimentally induced pressure to pursue PAp goals would undermine learning, learning strategies, and performance compared to a condition supporting the autonomous pursuit of PAp goals and a condition encouraging mastery goal pursuits. The results of previous experimental achievement-goal research has been mixed, with some researchers finding a mastery goal induction to result in superior performance (e.g., Graham and Golan 1991) and intrinsic motivation (e.g., Utman 1997) compared to a performance goal induction, whereas others have failed to replicate these findings (e.g., Elliot 2005). It would be interesting to explore whether PAp goals would differently predict outcomes depending on whether these PAp goals were communicated in an autonomy-supportive versus controlling way, much like the autonomous and controlled regulation of PAp goals yielded a different relation to the learning outcomes in this study (see also Spray et al. 2006).

Future research might also examine whether more classic personality factors that have received extensive attention within the achievement motivation tradition (i.e., the motive to succeed or to avoid failure; Atkinson 1957; Elliot 1999), and teachers' autonomy-supportive versus controlling styles (Reeve et al. 2004) are differentially related to the reasons underlying students' PAp goal pursuits. Although it has been shown within the hierarchical model of achievement motivation (Elliot and Church 1997) that both the motives to succeed and to avoid failure are predictive of the endorsement of PAp goals, we would expect them to be differentially predictive of the reasons underlying the pursuit of PAp goals, with the motive to succeed being associated with a more autonomous regulation of PAp goals and the motive to avoid failure being associated with a more controlled regulation of PAp goals. Finally, the current project focused on the reasons for PAp-goal pursuits, but it would be equally instructive to examine whether the reasons behind other achievement goals (see Dompnier et al. 2009 for initial steps) would also yield differential effects on learning outcomes.

\section{Conclusion}

Achievement goal theory has been a popular framework for understanding and predicting students' functioning in school. Whereas early researchers (e.g., Nicholls 1984; Ryan 1982) defined achievement goals in a way that includes the reasons for engaging in an achievement setting (e.g., ego-orientation), Elliot and colleagues (Elliot 1999, 2005; Elliot and Thrash 2001) have recently argued that achievement goals need to be defined only in terms of that the criteria for inferring competence, and that different reasons could motivate any particular goal. The present study shows that the underlying reasons for PAp goals matter more for optimal learning and performance than does the strength of the PAp goal per se. In line with selfdetermination theory, the present research shows that whereas autonomous or volitional regulation of PAp goals relates positively to adjustment and learning, controlled or pressured regulation of PAp goals relates to a more diminished set of outcomes. In our view, the differential pattern of results associated with the two types of regulations is striking and important both because it provides insight into when the pursuit of PAp goals is adaptive versus maladaptive and because it represents a conceptual advance in theorizing about the effects of PAp goals.

Acknowledgments We would like to thank Ellen Duyck for her fantastic help in collecting the data.

\section{References}

Accordino, D. B., Accordino, M. P., \& Slaney, R. B. (2000). An investigation of perfectionism, mental health, achievement and achievement motivation in adolescents. Psychology in the Schools, 37, 535-545.

Ames, C. (1992). Classrooms: Goals, structures, and student motivation. Journal of Educational Psychology, 84, 261-271.

Anderman, E. M., Griesinger, T., \& Westerfield, G. (1998). Motivation and cheating during early adolescence. Journal of Educational Psychology, 90, 84-93.

Assor, A., Vansteenkiste, M., \& Kaplan, A. (2009). Identified and introjection approach and introjection avoidance motivations in school and in sport: The limited benefits of self-worth strivings. Journal of Educational Psychology, 101, 482-497.

Atkinson, J. W. (1957). Motivational determinants of risk-taking behavior. Psychology Review, 64, 359-372.

Atkinson, J. W., \& Feather, N. T. (Eds.). (1966). A theory of achievement motivation. New York: Wiley.

Barron, K. E., \& Harackiewicz, J. M. (2001). Achievement goals and optimal motivation: Testing multiple goal models. Journal of Personality and Social Psychology, 80, 706-722.

Bentler, P. (1990). Comparative fit indexes in structural equation models. Psychological Bulletin, 107, 238-246. 
Bieling, P. J., Israeli, A., Smith, J., \& Antony, M. M. (2003). Making the grade: Behavioural consequences of perfectionism in the classroom. Personality and Individual Differences, 35, 163-178.

Blatt, S. J. (1995). The destructiveness of perfectionism. American Psychologist, 50, 1003-1020.

Boekaerts, M. (1997). Self-regulated learning: A new concept embraced by researchers, policy makers, educators, teachers, and students. Learning and Instruction, 7, 161-186.

Bollen, K. (1989). Structural equations with latent variables. New York: Wiley.

Brophy, J. (2005). Goal theorists should move on from performance goals. Educational Psychologist, 40, 167-176.

Browne, M., \& Cudeck, R. (1993). Alternative ways of assessing model fit. In K. Bollen \& J. Long (Eds.), Testing structural equation models (pp. 136-162). Newbury Park, CA: Sage.

Butler, R. (1992). What young people want to know when: Effects of mastery and ability goals on interest in different kinds of social comparisons. Journal of Personality and Social Psychology, 62, 934-943.

Ciani, K. D., Middleton, M. J., Summers, J. J., \& Sheldon, K. M. (2010). Buffering against performance classroom goal structures: The importance of autonomy support and classroom community. Contemporary Educational Psychology, 35, 88-99.

Darnon, C., Butera, F., Mugny, G., Quiamzade, A., \& Hulleman, C. S. (2009). "Too complex for me!": Why do performance-approach and performance-avoidance goals predict exam performance? European Journal of Psychology of Education, 24, 423-434.

De Bilde, J., Vansteenkiste, M., \& Lens, W. (2010). Underderstanding the association between future time perspective and selfregulated learning through the lens of self-determination theory. Learning and Instruction (in press).

Deci, E. L., \& Ryan, R. M. (1985). The general causality orientations scale: Self-determination in personality. Journal of Research in Personality, 19, 109-134.

Deci, E. L., \& Ryan, R. M. (2000). The "what" and "why" of goal pursuits: Human needs and the self-determination of behavior. Psychological Inquiry, 11, 227-268.

DeCoster, S., Vansteenkiste, M., \& Lens, W. (2007). Intrapersonal achievement goals: What is their conceptual status and what are their effects? Unpublished Research Report, University of Leuven.

Dompnier, B., Darnon, C., \& Butera, F. (2009). Faking the desire to learn: A clarification of the link between mastery goals and achievement. Psychological Science, 20, 939-943.

Dunkley, D. M., Blankstein, K. R., Masheb, R. M., \& Grilo, C. M. (2006). Personal standards and evaluative concerns dimensions of "clinical" perfectionism: A reply to Shafran et al. (2002, 2003) and Hewitt et al. (2003). Behaviour Research and Therapy, 44, 63-84.

Dweck, C. S. (1986). Motivational processes affecting learning. American Psychologist, 41, 1040-1048.

Dweck, C., \& Leggett, E. (1988). A social-cognitive approach to motivation and personality. Psychology Review, 95, 256-273.

Elliot, A. J. (1999). Approach and avoidance motivation and achievement goals. Educational Psychologist, 34, 169-189.

Elliot, A. J. (2005). A conceptual history of the achievement goal construct. In A. Elliot \& C. Dweck (Eds.), Handbook of competence and motivation. New York: Guilford Press.

Elliot, A. J., \& Church, M. A. (1997). A hierarchical model of approach and avoidance achievement motivation. Journal of Personality and Social Psychology, 72, 218-232.

Elliot, A. J., \& Harackiewicz, J. M. (1996). Approach and avoidance achievement goals and intrinsic motivation: A mediational analysis. Journal of Personality and Social Psychology, 70, 461-475.

Elliot, A. J., \& McGregor, H. A. (1999). Test anxiety and the hierarchical model of approach and avoidance achievement motivation. Journal of Personality and Social Psychology, 76, 628-644.

Elliot, A. J., \& McGregor, H. A. (2001). A $2 \times 2$ achievement goal framework. Journal of Personality and Social Psychology, 80, 501-519.

Elliot, A. J., McGregor, H. A., \& Gable, S. (1999). Achievement goals, study strategies, and exam performance: A mediational analysis. Journal of Educational Psychology, 91, 549-563.

Elliot, A. J., \& Moller, A. C. (2003). Performance-approach goals: Good or bad forms of regulation? International Journal of Educational Research, 39, 336-356.

Elliot, A. J., \& Murayama, K. (2008). On the measurement of achievement goals: Critique, illustration and application. Journal of Educational Psychology, 100, 613-628.

Elliot, A. J., \& Thrash, T. M. (2001). Achievement goals and the hierarchical model of achievement motivation. Educational Psychology Review, 13, 139-156.

Elliot, A. J., Zahn, I., \& Maier, M. J. (2008). Why are you doing this? The reasons behind performance approach goals may alter achievement outcomes. In Paper presented at the annual meeting of the American educational research association (AERA). New York.

Enns, M. W., Cox, B. J., \& Clara, I. (2002). Adaptive and maladaptive perfectionism: Developmental origins and association with depression proneness. Personality and Individual Differences, 33, 921-935.

Fox, J. (1991). Regression diagnostics. Thousand Oaks, CA: Sage.

Frost, R. O., Heimberg, R. G., Holt, C. S., Mattia, J. I., \& Neubauer, A. L. (1993). A comparison of two measures of perfectionism. Personality and Individual Differences, 14, 119-126.

Frost, R. O., Marten, P., Lahart, C., \& Rosenblate, R. (1990). The dimensions of perfectionism. Cognitive Therapy and Research, 14, 449-468.

Frost, R. O., Trepanier, K. L., Brown, E. J., Heimberg, R. G., Juster, H. R., Makris, G. S., et al. (1997). Self-monitoring of mistakes among subjects high and low in perfectionistic concern over mistakes. Cognitive Therapy and Research, 21, 209-222.

Graham, S., \& Golan, S. (1991). Motivational influences on cognition: Task Involvement, ego involvement, and depth of information processing. Journal of Educational Psychology, 83, 187-194.

Grzegorek, J. L., Slaney, R. B., Franze, S., \& Rice, K. G. (2004). Selfcriticism, dependency, self-esteem, and grade point average satisfaction among clusters of perfectionists and nonperfectionists. Journal of Counseling Psychology, 51, 192-200.

Hair, J. F., Anderson, R., Tatham, R. L., \& Black, W. C. (2006). Multivariate data analysis. Upper Saddle River, NJ: Prentice Hall.

Hamachek, D. E. (1978). Psychodynamics of normal and neurotic perfectionism. Psychology, 15, 27-33.

Hambleton, R. K. (1994). Guidelines for adapting educational and psychological tests: A progress report. European Journal of Psychological Assessment, 10, 229-244.

Harackiewicz, J. M., Barron, K. E., \& Elliot, A. J. (1997). Predictors and consequences of achievement goals in the college classroom: Maintaining interest and making the grade. Journal of Personality and Social Psychology, 73, 1284-1295.

Harackiewicz, J. M., Barron, K. E., \& Elliot, A. J. (1998). Rethinking achievement goals: When are they adaptive for college students and why? Educational Psychologist, 33, 1-21.

Harackiewicz, J. M., Barron, K. E., Pintrich, P. R., Elliot, A. J., \& Thrash, T. M. (2002). Revision of achievement goal theory: Necessary and illuminating. Journal of Educational Psychology, 94, 638-645.

Holmbeck, G. N. (1997). Toward terminological, conceptual, and statistical clarity in the study of mediators and moderators: 
Examples from the child-clinical and pediatric psychology literatures. Journal of Consulting and Clinical Psychology, 4, 599-610.

Hu, L., \& Bentler, P. M. (1999). Cutoff criteria for fit indexes in covariance structure analysis: Conventional criteria versus new alternatives. Structural Equation Modeling, 6, 1-55.

Jordan, A. E. (2001). College student cheating: The role of motivation, perceived norms, attitudes, and knowledge of institutional policy. Ethics and Behavior, 11, 233-247.

Joreskog, K., \& Sorbom, D. (1996). Lisrel 8 user's reference guide. Chicago: Scientific Software International.

Kaplan, A., \& Maehr, M. L. (2007). The contributions and prospects of goal orientation theory. Contemporary Educational Psychology, 19, 141-184.

Kenney-Benson, G. A., Pomerantz, E. M., Ryan, A. M., \& Patrick, H. (2006). Sex differences in math performance: The role of children's approach to schoolwork. Developmental Psychology, $42,11-26$.

Lacante, M., \& Lens, W. (2005). Nederlandstalige aanpassing van de Weinstein-Palmer LASSI-scales [Dutch adaptation of the Weinstein-Palmer LASSI-scales]. Unpublished research paper, University of Leuven, Belgium.

Lau, S., \& Nie, Y. (2008). Interplay between personal goals and classroom goal structures in predicting student outcomes: A multilevel analysis of person-context interactions. Journal of Educational Psychology, 100, 15-29.

Matos, L., Lens, W., \& Vansteenkiste, M. (2007). Achievement goal orientations, learning strategies and language achievement among Peruvian high school students. Psychologica Belgica, $47,51-70$

McClelland, D. C., Atkinson, J. W., Clark, R. A., \& Lowell, E. L. (1953). The achievement motive. New York: Appleton-CenturyCrofts.

Midgley, C., Kaplan, A., \& Middleton, M. (2001). Performanceapproach goals: Good for what, for whom, under what circumstances? Journal of Educational Psychology, 93, 77-86.

Miles, J., \& Shevlin, M. (2001). Applying regression and correlation. Thousand Oaks, CA: Sage.

Mills, J. S., \& Blanstein, K. R. (2000). Perfectionism, intrinsic and extrinsic motivation, and motivated strategies for learning: A multidimensional analysis of university students. Personality and Individual Differences, 29, 1191-1204.

Miquelon, P., Vallerand, R. J., Grouzet, F. M. E., \& Cardinal, G. (2005). Perfectionism, academic motivation, and psychological adjustment: An integrative model. Personality and Social Psychology Bulletin, 31, 913-924.

Murayama, K., \& Elliot, A. J. (2009). The joint influence of personal achievement goals and classroom goal structure on achievementrelevant outcomes. Journal of Educational Psychology, 101, 432-447.

Newstead, S. E., Franklyn, S. A., \& Armstead, P. (1996). Individual differences in cheating. Journal of Educational Psychology, 88, 229-241.

Nicholls, J. G. (1984). Achievement motivation: Conceptions of ability, subjective experience, task choice, and performance. Psychology Review, 91, 328-346.

Ommundsen, Y., Roberts, G. C., Lemyre, P.-N., \& Miller, B. W. (2005). Peer relationships in adolescent competitive soccer: Associations to perceived motivational climate, achievement goals and perfectionism. Journal of Sports Sciences, 23, 977-989.

Pintrich, P. R., \& DeGroot, E. V. (1990). Motivational and selfregulated learning. Journal of Educational Psychology, 82, 33-40.

Reeve, J., \& Deci, E. L. (1996). Elements of the competitive situation that effect intrinsic motivation. Personality and Social Psychology Bulletin, 22, 24-33.
Reeve, J., Deci, E. L., \& Ryan, R. M. (2004). Self-determination theory: A dialectical framework for understanding socio-cultural influences on student motivation. In S. Van Etten \& M. Pressley (Eds.), Big theories revisited (pp. 31-60). Greenwich, CT: Information Age Press.

Rice, K. G., \& Slaney, R. B. (2002). Clusters of perfectionists: Two studies of emotional adjustment and academic achievement. Measurement and Evaluation in Counseling and Development, $35,35-48$.

Ryan, R. M. (1982). Control and information in the intrapersonal sphere: An extension of cognitive evaluation theory. Journal of Personality and Social Psychology, 43, 450-461.

Ryan, R. M., \& Connell, J. P. (1989). Perceived locus of causality and internalization: Examining reasons for acting in two domains. Journal of Personality and Social Psychology, 57, 749-761.

Ryan, R. M., \& Deci, E. L. (2000). Self-determination theory and the facilitation of intrinsic motivation, social development and wellbeing. American Psychologist, 55, 68-78.

Satorra, A., \& Bentler, P. M. (1994). Corrections to test statistics and standard errors in covariance structure analysis. In A. Von Eye \& C. C. Clogg (Eds.), Latent variables analysis: Applications for developmental research (pp. 399-419). Thousand Oaks, CA: Sage.

Schunk, D. H., \& Zimmerman, B. J. (Eds.). (1994). Self-regulation of learning and performance: Issues and educational applications. Hillsdale, NJ: Lawrence Erlbaum.

Senko, C., Durik, A. M., \& Harackiewicz, J. (2008). Historical perspectives and new directions in achievement goal theory: Understanding the effects of mastery and performance-approach goals. In J. Y. Shah \& W. L. Gardner (Eds.), Handbook of motivation science (pp. 100-113). New York: Guilford Press.

Shafran, R., \& Mansell, W. (2001). Perfectionism and psychopathology: A review of research and treatment. Clinical Psychology Review, 21, 879-906.

Sheldon, K. M. (2002). The self-concordance model of healthy goalstriving: When personal goals correctly represent the person. In E. L. Deci \& R. M. Ryan (Eds.), Handbook of self-determination research (pp. 65-86). Rochester, NY: The University of Rochester Press.

Sheldon, K. M., \& Kasser, T. (1995). Coherence and congruence: Two aspects of personality integration. Journal of Personality and Social Psychology, 68, 531-543.

Soenens, B., Vansteenkiste, M., Luyten, P., Duriez, B., \& Goossens, L. (2005). Maladaptive perfectionistic self-representations: The mediational link between psychological control and adjustment. Personality and Individual Differences, 38, 487-498.

Spray, C. M., Wang, C. K. J., Biddle, S. J., \& Chatzisarantis, N. L. D. (2006). Understanding motivation in sport: An experimental test of achievement goal and self-determination theories. European Journal of Sport Sciences, 6, 43-51.

Steiger, J. H. (1990). Structural model evaluation and modification: An interval estimation approach. Multivariate Behavioral Research, 25, 173-180.

Stoeber, J., Uphill, M. A., \& Hotham, S. (2009). Predicting race performance in triathlon: The role of perfectionism, achievement goals and personal goal setting. Journal of Sport and Exercise Psychology, 31, 211-245.

Urdan, T., \& Mestas, M. (2006). The goals behind performance goals. Journal of Educational Psychology, 98, 354-365.

Utman, C. H. (1997). Performance effects of motivational state: A meta-analysis. Personality and Social Psychology Review, 1, 170-182.

Vansteenkiste, M., Lens, W., Dewitte, S., De Witte, H., \& Deci, E. L. (2004). The 'why' and 'why not' of job search behavior: Their relation to searching, unemployment experience and well-being. European Journal of Social Psychology, 34, 345-363. 
Vansteenkiste, M., Matos, L., Lens, W., \& Soenens, B. (2007). Understanding the impact of intrinsic versus extrinsic goal framing on exercise performance: The conflicting role of task and ego involvement. Psychology of Sport and Exercise, 8, 771-794.

Vansteenkiste, M., Niemiec, C., \& Soenens, B. (2010). The development of the five mini-theories of self-determination theory: An historical overivew, emerging trends, and future directions. In T. Urdan \& S. Karabenick (Eds.), Advances in motivation and achievement, vol. 16: The decade ahead (pp. 105-166). UK: Emerald Publishing.
Vansteenkiste, M., Zhou, M., Lens, W., \& Soenens, B. (2005). Experiences of autonomy and control among Chinese learners: Vitalizing or immobilizing? Journal of Educational Psychology, 97, 468-483.

Weinstein, C. E., \& Palmer, D. R. (1987). Learning and study strategies inventory (LASSI). Clearwater: $\mathrm{H} \& \mathrm{H}$ Publishing Company.

Wolters, C. A. (2004). Advancing achievement goal theory: Using goal structures and goal orientations to predict students' motivation, cognition, and achievement. Journal of Educational Psychology, 96, 236-250. 